\title{
Incidence of piperacillin-specific drug antibody in patients with and without piperacillin
}

\author{
Zhiwei Liu ${ }^{1}$, Zhen Cheng ${ }^{2}, \mathrm{Na} \mathrm{Ma}^{3}$,Baolan $\mathrm{Hao}^{4}$, Dan $\mathrm{Xu}^{5}$, Yetao Han ${ }^{1}$, Mengsi Hu ${ }^{1}$, Rui Wang ${ }^{1}$, \\ Daowang Fan ${ }^{6}$, Meiying Rao ${ }^{7 *}$ \\ ${ }^{1}$ Department of Blood Transfusion, Sir Run Run Shaw Hospital, School of Medicine, Zhejiang University, Hangzhou 310016, Zhejiang, China; \\ ${ }^{2}$ International Healthcare Center, Sir Run Run Shaw Hospital, School of Medicine, Zhejiang University, Hangzhou 310016, Zhejiang, China; \\ ${ }^{3}$ Department of Blood Transfusion, Cancer Hospital of Yunnan Province, Kunming 650106, Yunnan, China; \\ ${ }^{4}$ Blood Transfusion Department, Affiliated Tumor Hospital of Zhengzhou University, Zhengzhou 450008, Henan, China; \\ ${ }^{5}$ Jiangsu LIBO Medicine Biotechnology Co., Ltd., Jiangyin 214434, Jiangsu, China; \\ ${ }^{6}$ Blood Group Lab, Beijing Red Cross Blood Center, Beijing 100088, China; \\ ${ }^{7}$ Department of Blood Transfusion, the Second Affiliated Hospital of Nanchang University, Nanchang 330006, Jiangxi, China.
}

\begin{abstract}
The aim of this study was to investigate the incidence of piperacillin-specific drug antibody in patients with and without piperacillin. Piperacillin antibody was detected by piperacillin-induced hemolysis test kit. Three hundred samples were collected from patients who had been treated with piperacillin within 3 months; and 222 samples were from patients who were treated with other antibiotics; and a normal group of 120 samples was from healthy blood donors. The results showed that the positive rates in the piperacillin group, the other antibiotic group and the control group were $11.33 \%, 4.95 \%$ and $1.67 \%$, respectively, which was statistically significant. The antibody titer was from 2 to 64 . The antibody titer of the piperacillin group was significantly higher than that of the other two groups. The study suggests that piperacillin is able to induce piperacillin-specific drug antibody and drug antibody detection is useful for maintaining the safety of medication.
\end{abstract}

Keywords: piperacillin-specific drug antibody, piperacillin sensitized RBC, drug safety

\section{INTRODUCTION}

The top five rankings of China's adverse drug reaction monitoring reports in 2017 were antimicrobials, cardiovascular drugs, antineoplastic agents, water-electrolyte, acid-base balance drugs and digestive system drugs. The reported proportion of antimicrobial drugs was highest (43.9\%). The top 3 varieties

*Correspondence to: Meiying Rao, Department of blood transfusion, The Second Affiliated Hospital of Nanchang University, No.1 Minde Road, Nanchang 330006,Jiangxi, China. Tel: 0086-18970074965, Email: rmy_nanchang@126.com. of serious adverse reactions / incident reports were all antimicrobials ${ }^{[1]}$. Chinese domestic and wider international adverse drug reaction / incident report forms however, only focus on the causality and severity of side effects caused by drugs, and diagnostic tools and preventive measures for laboratory diagnostic drug antibodies are still lacking ${ }^{[2]}$.

Piperacillin (a semi-synthetic penicillin) is used to treat respiratory tract, gastrointestinal tract and urinary tract infections, as its suitability for treating sensitive strains of certain gram-negative bacteria and anaerobic bacteria ${ }^{[3-4]}$. In the past half century, the use of piperacillin at home and abroad has reached the top five of all antibacterial penicillin injections. 
Piperacillin is the third most common drug that causes drug-induced hemolytic anemia, of which cefotetan is the most common and ceftriaxone is the second one ${ }^{[5]}$. The mechanism of piperacillininduced immune hemolytic anemia is an antibody/ hapten immune response, in which piperacillin binds to surface protein of erythrocyte-cell membranes to form the hapten/protein complex. Penicillin-induced hemolysis, which usually takes place in the extravascular space, usually occurs approximately 10 days after penicillin treatment ${ }^{[6-7]}$. In this study, the piperacillin induced hemolysis kit was used to analyze the incidence of piperacillin antibody in patients with and without piperacillin.

\section{MATERIALS AND METHODS}

\section{Blood samples}

A total of 642 blood samples were collected from the Sir Run Run Shaw Hospital affiliated to Zhejiang University from January 2017 to May 2018, including 300 samples from patients who had been treated with piperacillin within 3 months (piperacillin group), 222 samples from patients who were treated with other antibiotics (other antibiotic group) and the normal group of 120 samples from healthy blood donors (control group). Whole blood was taken and plasma was separated for testing. The penicillin skin test was negative before the patient was given the drug. The study was approved by the Human Research Ethics Committee of the hospital and had informed consent from the patients themselves.

\section{Drug antibody detection}

A piperacillin-induced hemolysis test kit was used as the experimental reagent (Jiangsu Zhongji Wantai Bio-Pharmaceutical Co., Ltd., batch number 1803002, 1803004, 18040017, 18050038, 18060025). The main components of the kit were drug-sensitized red blood cells, micro-column gel cards containing anti-human IgG special for drug antibody detection and positive and negative control (where the manufacturer provides the sample with or without piperacillin antibody).

According to the test kit's instruction manual, the plasma to be tested was diluted 1:19 with physiological saline. Each test consisted of: (1) Positive control: positive control $50 \mu \mathrm{L}+$ drug-sensitized red blood cells $25 \mu \mathrm{L}$; (2) Negative control: negative control $50 \mu \mathrm{L}+$ drug-sensitized red blood cells 25 $\mu \mathrm{L}$; (3) Self-control: Plasma to be tested $50 \mu \mathrm{L}$ +unsensitized red blood cells $25 \mu \mathrm{L}$; (4) Drug antibody detection: Plasma to be tested $50 \mu \mathrm{L}+\mathrm{drug}$-sensitized red blood cells $25 \mu \mathrm{L}$.

Further instructions were to: take the drug antibody detection reagent card, add the cells and plasma respectively, mix well, incubate for $1 \mathrm{~h}$ at $(37 \pm 1)^{\circ} \mathrm{C}$ in a reagent card incubator and then centrifuge for $5 \mathrm{~min}$ $(900 \mathrm{r} / \mathrm{min} \times 2 \mathrm{~min}$ and $1,500 \mathrm{r} / \mathrm{min} \times 3 \mathrm{~min})$. The results were observed and recorded within $30 \mathrm{~min}$.

\section{Statistical analysis}

All the statistical analyses were conducted by using SPSS version 19.0. Comparison results with a 2 -sided. $P$ value $<0.05$ were considered statistically significant.

\section{RESULTS}

\section{Piperacillin antibody detection}

The positive rates of piperacillin antibody were: 34 cases in the piperacillin group: $11.33 \%$ (34/300); 11 cases in the other antibiotics group: $4.95 \%$ $(11 / 222)$; and 2 cases in the normal control group: $1.7 \%(2 / 120$, Table 1).

Table 1 The detection of piperacillin antibody

\begin{tabular}{ccccccc}
\hline Groups & $n$ & $\begin{array}{c}\text { Positive } \\
(n)\end{array}$ & $\begin{array}{c}\text { Weak } \\
\text { positive }(n)\end{array}$ & $\begin{array}{c}\text { Negative } \\
(n)\end{array}$ & $\begin{array}{c}\text { Positive } \\
\text { rate(\%) }\end{array}$ & $P$ value \\
\hline $\begin{array}{c}\text { Piperacillin } \\
\text { group }\end{array}$ & 300 & 20 & 14 & 266 & 11.33 & 0.008 \\
$\begin{array}{c}\text { Other antibiotic } \\
\text { group }\end{array}$ & 222 & 4 & 7 & 211 & 4.95 & \\
\begin{tabular}{c} 
Control group \\
\hline
\end{tabular} & 120 & 0 & 2 & 118 & 1.67 & \\
\hline
\end{tabular}

\section{Piperacillin antibody titer}

The antibody titers of all 47 positive samples were determined(Table 2). In the piperacillin group, there were 14 samples with an antibody titer of less than 2 , 8 samples with an antibody titer of 2,4 samples with titer of 4, 3 samples with titer of 8,2 samples with titer of 16, 2 samples with titer of 32 and 1 sample with a titer of 64, respectively; In the other antibiotic group, there were 7 samples with an antibody titer of less than 2, 3 samples with antibody titer of 2 , and 1 samples with an antibody titer of 4 , respectively. In the control group there were only 2 samples with an antibody titer less than 2 .

\section{Age analysis}

The observation objects' ages were analyzed separately. In piperacillin group, the positive antibody rates of 18 55 age and > 55 age were $10.6 \%$ and $11.9 \%$, respectively; in other antibiotic group, 
Table 2 The titers of piperacillin antibody (n)

\begin{tabular}{crrrrrrrr}
\hline \multirow{2}{*}{ Groups } & \multicolumn{7}{c}{ Drug antibody titer of piperacillin } \\
\cline { 2 - 10 } & Total & $<2$ & 2 & 4 & 8 & 16 & 32 & 64 \\
\hline Piperacillin group & 34 & 14 & 8 & 4 & 3 & 2 & 2 & 1 \\
Other antibiotic group & 11 & 7 & 3 & 1 & 0 & 0 & 0 & 0 \\
Control group & 2 & 2 & 0 & 0 & 0 & 0 & 0 & 0 \\
\hline
\end{tabular}

the antibody positive rates of 18 55 age and > 55 age were $3.9 \%$ and $5.8 \%$, respectively. No blood donors over 55 years old were in control group (Table 3).

Table 3 Sample age statistics

\begin{tabular}{ccccccc}
\hline Groups & $n$ & $\begin{array}{c}\text { Positive } \\
(n)\end{array}$ & $\begin{array}{c}\text { Weak } \\
\text { positive }(n)\end{array}$ & $\begin{array}{c}\text { Negative } \\
(n)\end{array}$ & $\begin{array}{c}\text { Positive } \\
\text { rate }(\%)\end{array}$ & $P$ value \\
\hline $\begin{array}{c}\text { Piperacillin } \\
\text { group }\end{array}$ & 300 & 20 & 14 & 266 & 11.33 & 0.008 \\
$\begin{array}{c}\text { Other antibiotic } \\
\text { group }\end{array}$ & 222 & 4 & 7 & 211 & 4.95 & \\
\begin{tabular}{c} 
Control group \\
\hline
\end{tabular} & 120 & 0 & 2 & 118 & 1.67 & \\
\hline
\end{tabular}

\section{DISCUSSION}

In 2017, the National Adverse Drug Reaction Monitoring Network received 433,000 new and serious adverse drug reaction / incident reports, of which 55,000 were serious. Among the suspected drugs, the first three categories of chemical cases were anti-infective drugs $(42.3 \%)$, cardiovascular system drugs $(10.0 \%)$, and cancer treatment drugs $(7.3 \%)$, respectively. Anti-infective drugs were by far the main cause of adverse drug reactions ${ }^{[1]}$. The current monitoring system for adverse drug reactions mainly observes and tests the severity of adverse reactions and looks for the causes, but lacks direct laboratory diagnostic tools, and therefore lacks preventive methods ${ }^{[2]}$.

Drug-induced immune hemolysis can be caused by different mechanisms. It is generally believed that there are three main mechanisms: (1) The drug is converted into a complete antigen after binding to red blood cells, and the whole immunogenicity is expressed, and the immune cells recognize the drug antigen to destroy the cells; (2) Antibodies in plasma and drugs form immune complexes that are adsorbed on cells and produce antibodies against drugs or their metabolites; (3) The drug only binds loosely to the cells, and the cell does not produce drug antibodies, but produces autoantibodies. A penicillin-like drug represented by piperacillin, which induces drug antibody production, has a hapten mechanism. Piperacillin as a hapten covalently binds to proteins on the erythrocyte membrane, stimulating the body to produce antibodies. After the production of antibodies, the continued use of large amounts of drugs leads to the production of drug-induced immune hemolysis. ${ }^{[8-11]}$

Leger RM et al. ${ }^{[12]}$ used piperacillin-sensitized red blood cells to detect the plasma of patients, found a certain proportion of blood donor plasma and patient plasma agglutinated with piperacillin-sensitized red blood cells, and piperacillin antibody was detected in some patient plasma. In China, domestic research on drug antibodies is in its infancy, and lacks a systematic approach. According to one report, 8 cases of anti-penicillin antibodies were detected in 181 samples, among which 4 cases had obvious hemolytic reaction. In addition, another report published data from 327 antibiotic drug treatments, showing 13 cases having anti-drug antibodies, 4 of which had an obvious hemolytic reaction ${ }^{[13-14]}$.

In this study, the piperacillin induced hemolysis kit was used to detect piperacillin antibody in patients' plasma by using drug sensitized erythrocyte in microcolumn gel. Among them, 120 participants represented healthy blood donors, from which the positive antibody rate was $1.7 \%$; 222 cases of patients were treated with other antibiotics, resulting in a positive rate of $4.9 \%$; 300 patients were treated with piperacillin, at a positive rate of $11.3 \%$. The finding was that drug antibodies produced after drug treatment may increase the risk of adverse reactions to subsequent antibiotic treatment. In the case of adverse reactions, false negative cases is common: the penicillin skin test is negative, but there remains a large number of drug antibodies in patients plasma. When a patient see a doctor, the clinician should ask about the history of allergies and family history in detail. When using piperacillin, skin tests and drug antibody tests should be performed at the same time, which can effectively reduce adverse reactions caused by piperacillin.

The antibody titer of the piperacillin group was significantly higher than that of the other groups. There were 8 cases in which the antibody titer of the piperacillin group was more than 8 , but the antibody titers of the other two groups were all less. Positive results in other antibiotic groups may be due to drug cross-reactivity. Piperacillin belongs to the penicillin drug group. Antibiotic drugs have similar molecular structures and their immunogenicity is relatively close, so cross-reaction may occur between drugantibodies and drugs. The positive result in the control group may have been due to the changes in the erythrocyte membrane caused by piperacillin, and the non-immune adsorption of protein on the erythrocyte membrane, causing the test results to be positive, so the titer was relatively low.

In this study, statistical analysis was performed 
on 642 samples. The results indicate that as patients age increases, the likelihood of using antibiotics increases, and subsequently the incidence of drug antibodies also increases. It is possible that only the first piperacillin is sensitized, or that a large quantity of piperacillin will activate the immune cells to produce a large number of piperacillin antibodies, leading to serious hemolysis and serious drug-induced immune hemolytic anemia. This means that different individuals will have different antibody piperacillin titers. Piperacillin is commonly used in patients with cystic fibrosis screening, however anti-piperacillin antibodies should also be taken into consideration whenever these patients develop hemolytic anemia or test positive in the direct antiglobulin test.

Commonly used drug-based antibody detection methods include the traditional test tube method, micro-column gel method, ELISA method, flow cytometry, and so on. The microcolumn gel antihuman globulin method is widely used in the detection of irregular blood group antibodies. The method for the detection of drug antibodies was established by combining the erythrocyte sensitizing technique with the microcolumn gel detection system. This can effectively detect piperacillin drug antibodies in blood samples and provide a judgment basis for clinical treatment and the safe use of transfused blood.

\section{Acknowledgments and funding}

This work was supported by the grant of Medical Science Research Foundation of Zhejiang.

\section{References}

[1] State Administration for Market Regulation. National annual report on adverse drug reaction monitoring
(2017) [J]. Chinese Drug Evaluation, 2018,2: 154-160.

[2] Chen JY, Yuan L, Yang Y. Conception of establishing computerized quality assessment system of adverse drug reaction/event report in China[J]. Chinese Drug Alert,2017,14(11):674-679

[3] Pan HW. Clinical efficacy of piperacillin and ampicillin in the treatment of community-acquired pneumonia[J]. Electron J Clin Med, 99(4):19551-19553

[4] Ye LQ, Cai T, Jin YH, et al. Clinical study on extending infusion time of piperacillin/tazobactam in treatment of gram-negative bacteria I nfection[J]. Chin J Nosocomiol,2011,21(16):3476-3479

[5] Garratty G. Drug-induced immune hemolytic anemia[J]. Clin Adv Hematol Oncol,2009,8:73-79

[6] Petz LD, Fudenber HH. Coombs-positive hemolytic anemia caused by penicillin administration[J]. $N$ Engl $J$ Med,1966,274(4):171-178

[7] Jackson FN, Jaffe JP. Fatal penicillin-induced hemolytic-anemia[J]. JAMA, 1979,242(21):2286-2287

[8] Arndt PA, Garratty G, Hill J, et al. Two cases of immune hemolytic anemia, associated with anti-piperacillin, detected by the "immune complex" method[J]. Vox Sang, 2002, 83:273-278.

[9] Petz LD. Drug-induced autoimmune hemolytic anemia[J]. Transfus Med Rev, 1993, 7:242-254.

[10] Petz LD, Mueller-Eckhardt C. Drug-induced immune hemolytic anemia[J]. Transfusion, 1992, 32:202-204.

[11] Salama A. Drug-induced immune hemolytic anemia[J]. Expert Opin Drug Saf, 2009, 8:73-79.

[12] Leger RM, Arndt PA, Garratty G. Serological studies of piperacillin antibodies[J]. Transfusion, 2008, 48(11): 2429-2434

[13] Yang QX, Zhu ZY. Detection of anti-antibiotic antibodies by drug-coated red blood cells[J]. Modern Immunology, 2010(3):243-247

[14] Cao S. Microcolumn gel immunoassay for detection of anti-antibiotic antibodies in blood of patients[J]. Chinese Community Physician Li,2012,14(25):256-257

(Received 09 November 2018, Revised 08 December 2018, Accepted 17 December 2018) 